\title{
Proposição de um modelo baseado em Customer Lifetime Value para a análise de melhorias no sistema produtivo
}

\author{
Proposition of a Customer Lifetime Value model \\ to evaluate production system improvements
}

\author{
Luís Felipe Riehs Camargo' \\ Guilherme Luis Roehe Vaccaro'
}

\begin{abstract}
Resumo: Este artigo propõe um modelo para analisar melhorias no sistema produtivo à luz do valor do cliente para a empresa. Para isso, apresenta um modelo que agrega variáveis advindas do processo produtivo, do mercado consumidor e de métricas do marketing. Os três pilares de sustentação do modelo são os clientes, com suas necessidades e percepções em relação aos produtos e serviços; as características atribuídas pela produção; e as regras para análise do impacto da adoção de diferentes melhorias no sistema produtivo sobre o valor vitalício dos clientes atuais (CLV). Além disso, um modelo de escolha discreta (logit) é proposto como o integrador entre a produção e o marketing. O método de pesquisa utilizado foi composto de quatro etapas: levantamento de referencial; construção do modelo conceitual; aplicação em um caso; análise e discussão. Como resultados, observa-se que, havendo disponibilidade de informações e tempo para sua condução, o modelo contribui para a identificação de melhorias que contemplem simultaneamente a visão de produção e a de sustentabilidade financeira.
\end{abstract}

Palavras-chave: Modelagem. Melhorias. Sistema produtivo. CLV. Logit.

\begin{abstract}
This paper aims to propose a model to investigate the effect of improvements on the productive system on the organization considering customer value. The model proposed combine variables representing the productive process, market, and marketing metrics. It consists of three fundamental elements: consumers and their needs and perceptions of products and services; characteristics of the productive system; and rules to analyze the impact of adoption of different improvements on the productive system. Customer lifetime value (CLV) was measured and a discrete choice model (logit) is proposed as an integrator between production and marketing perspectives. The research methodology consisted of four steps: referential research, conceptual modeling, application in a particular case, and results analysis. As a result, it is observed that upon availability of information and time, it contributes to the identification of improvements involving both involving production vision and financial sustainability.
\end{abstract}

Keywords: Modeling. Productive. System improvement. Customer lifetime value. Logit.

\section{Introdução}

As decisões tomadas sobre investimentos no processo produtivo visam, tipicamente, a elevar a eficiência do processo ou a reduzir os custos de produção. $\mathrm{O}$ aumento significativo da concorrência tem forçado importantes mudanças no âmbito organizacional e na gestão da produção. Neste sentido, um amplo leque de conceitos e modelos de gestão surgiu para moldar os sistemas de produção modernos, destacando-se: o Sistema Toyota de Produção (STP), a Produção Enxuta (Lean Manufacturing), o Controle de Qualidade Total (TQC), a Teoria das Restrições (TOC), a reengenharia de processos, os sistemas integrados de gestão (MRPII, ERP, SCM, etc.), entre outros (ANTUNES JUNIOR et al., 2008). A massificação do movimento da Qualidade Total, observada na década de 1990 e no início do século XXI, em particular, fez com que inúmeras empresas adotassem as mais variadas ferramentas de melhoria da produção, diminuindo o valor dessa dimensão enquanto diferencial competitivo.

Quando determinado tipo de método é usado de forma ampla por muitos concorrentes, o diferencial não reside mais em sua utilização para melhorar a eficiência ou a eficácia do processo, mas em sua eficácia para a sustentabilidade e o crescimento da empresa como um todo. Sob esse prisma, a análise

\footnotetext{
Programa de Pós-graduação em Engenharia de Produção e Sistemas, Universidade do Vale do Rio dos Sinos - UNISINOS, Av. Unisinos, 950, Bairro Cristo Rei, São Leopoldo - RS, Brasil, CEP 93022-000, E-mails: feliperiehs@ pop.com.br; guilhermev@unisinos.br
} 
de qual ferramenta utilizar e qual processo produtivo melhorar passa a ser foco de decisões estratégicas corporativas (e, portanto, multidepartamentais). Um decisor deveria levar em conta o impacto das decisões tomadas frente aos clientes, já que são eles que pagam pelos produtos e serviços gerados pelo sistema produtivo e, portanto, efetivamente garantem o sucesso de uma ação de melhoria definida no ambiente interno da organização. Dessa forma, as manifestações das preferências dos clientes podem ser elementos importantes na priorização das melhorias no processo produtivo. Este artigo apresenta um modelo conceitual que visa a contribuir na obtenção de informações para auxiliar as decisões de investimentos em melhorias no processo produtivo no contexto descrito anteriormente.

O artigo propõe-se, com essa contribuição, a responder à seguinte questão: Como avaliar as alternativas de investimentos em melhorias em um processo produtivo para competir em requisitos solicitados pelo mercado consumidor atendido por uma organização? O modelo fornece os instrumentos necessários para avaliar diferentes técnicas e ferramentas de acordo com seu impacto no valor presente das receitas e despesas ao longo de toda a vida dos clientes.

Dado que, em um contexto industrial ou de serviços, há diferentes clientes, diversos requisitos e diversas técnicas de melhoria de processo produtivo, como comparar tais opções? Por exemplo, algumas opções de técnicas, como a troca rápida de ferramentas (TRF) e a mudança de layout propostas pelo STP, e o mecanismo tambor-pulmão-corda (TPM) sugerido pela TOC reduzem a deficiência imposta pelo processo quanto ao tempo de entrega. Entretanto, este é apenas um dos diversos requisitos valorizados pelos clientes de uma empresa. $\mathrm{O}$ artigo busca mostrar que tal comparação pode ser feita a partir de uma mesma métrica: o impacto no valor vitalício do cliente, medido através da comparação de receitas e despesas esperadas deste cliente nos diferentes cenários de melhorias.

O modelo proposto para responder o problema apresentado avalia conjuntamente os requisitos do mercado, o processo produtivo e a previsão de geração de receitas, para diferentes cenários de melhorias no processo produtivo. Para tanto, são elementos de entrada do modelo: as prioridades estabelecidas pelos clientes; a análise de capacidade do processo produtivo para identificar os gargalos que limitam as prioridades dos atributos destacados pelos clientes e uma formalização matemática do link entre os gargalos; as eventuais multas ocasionadas pelo não cumprimento de acordos contratuais; a probabilidade de retorno usando a teoria probabilística de modelos logit de escolha, e a métrica Customer Lifetime Value (CLV). A utilização da métrica CLV considerando parâmetros do sistema produtivo foi apresentada por
Niraj, Gupta e Narasimhan (2001), os quais integraram variáveis extraídas da cadeia de suprimentos ao modelo CLV.

Além desta introdução, este artigo está organizado de forma a apresentar uma breve revisão teórica visando a embasar o modelo, o modelo proposto e as considerações sobre o cálculo das informações requeridas para sua operacionalização. Na sequência, encontram-se a aplicação do modelo, as considerações finais e, por fim, as conclusões do artigo.

\section{Referencial teórico}

Esta seção destaca pesquisas localizadas na literatura nacional e internacional sobre o tema deste artigo, apresentando também as bases teóricas do modelo proposto.

Hallgren e Olhager (2006) propuseram um método para quantificar diferentes estratégias de manufatura, com base nos requisitos do mercado, nas capacidades da manufatura, nas categorias de decisão de ações na manufatura e em modelos quantitativos. Entretanto, o modelo apresentado por esses pesquisadores não aborda a discussão da escolha das melhores ferramentas para atingir os objetivos estratégicos traçados por uma dada organização. Rivera e Chen (2007) sugerem o Cost-Time Profile (CTP) para avaliar o impacto esperado em mudanças no processo produtivo, pressupondo que a dimensão tempo em manufatura é o principal fator para a determinação do custo. No entanto, limitam-se a abordar pontos de vista distintos do marketing ou da produção, desconsiderando as contribuições de sua interação. $\mathrm{Ou}$ seja, esses autores propõem-se apenas a identificar os requisitos de mercado e a ajustar o processo para refletir em melhorias nos itens identificados. Já, Hess e Lucas (2004) avaliam o equilíbrio de investimento nas duas áreas: marketing e produção, assumindo que são perspectivas separadas. Porém, não discutem como avaliar o impacto das melhorias na produção nas preferências dos clientes de maneira integrada. Segundo esses autores, melhorias na produção significam "to do the thing right" e melhorias no marketing significam "to do the right thing", propondo um modelo para alocação ótima de investimentos em marketing e produção separadamente. No presente artigo, propõe-se que é possível uma abordagem integrada, ou seja, "to do the right thing right".

O modelo proposto para atender o problema de pesquisa apresentado demanda o uso de diversos métodos e técnicas, que devem ser estruturados e interligados de forma a viabilizar a construção e a aplicação do modelo. $\mathrm{O}$ modelo busca integrar o sistema de produção, o mercado com suas preferências e a sustentabilidade financeira, conforme ilustrado na Figura 1.

Hauser (1993) salienta a importância das informações advindas dos consumidores na elaboração de estratégias, no auxílio à tomada de decisão e no 


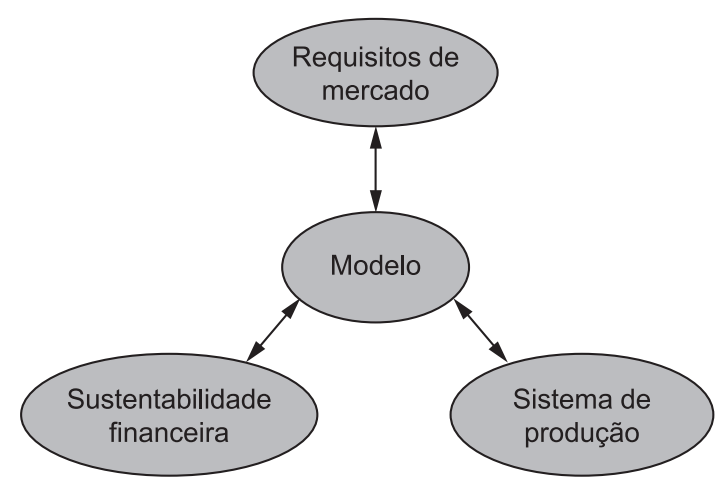

Figura 1. Integração sugerida.

desenvolvimento de novos produtos. Gupta e Zeithaml (2006) afirmam que, sem clientes, as empresas não possuem receitas nem lucros e, assim, não apresentam valor de mercado. Como pode ser observado na Figura 1, o mercado consumidor é um dos pilares do modelo, justamente por agregar as informações de preferências dos consumidores e o grau de importância nos requisitos que garantem a manutenção ou a ampliação dos negócios com a empresa nos produtos e serviços oferecidos. Hauser (1993) analisou diversos métodos para o desenvolvimento de novos produtos por meio das preferências apontadas pelos clientes, mas sem considerar os aspectos produtivos necessários para atender a tais preferências; diferentemente, no modelo proposto, essas informações são utilizadas para analisar melhorias no processo produtivo.

Hayes et al. (2008) afirmam que investimentos no sistema produtivo são uma fonte poderosa de vantagem competitiva. Isso denota a importância atribuída ao processo produtivo e às informações do mercado. Para o sucesso dos investimentos no sistema de produção, os requisitos apontados pelo mercado consumidor devem ser traduzidos adequadamente para uma linguagem que o processo produtivo compreenda sem perder as informações. O Quality Function Deployment (QFD) supre essa necessidade de transformar os atributos de preferência do mercado consumidor em parâmetros do sistema produtivo, segundo Cheng e Mello Filho (2007).

O QFD é um processo estruturado e disciplinado que permite identificar requisitos e preferências dos clientes nos estágios de desenvolvimento e implementação do produto (HAUSER, 1993; CHENG; MELLO FILHO, 2007). Esta técnica é adequada para interligar o que o cliente deseja com aquilo que o sistema de produção pode oferecer.

Uma vez obtidos os requisitos dos clientes, é necessário definir, a partir deles, os objetivos da manufatura. Por exemplo, se o requisito entrega confiável for eleito como prioritário pelos clientes, a operação deverá direcionar seus esforços à dimensão confiabilidade (FRANCO, 2001).
Segundo Godinho Filho e Fernandes (2005) e Hayes et al. (2008), existem trade offs que tornam impossível o atendimento de todos os objetivos da manufatura ao mesmo tempo. Quando os clientes requisitam produtos e serviços livres de erros e com baixo preço, não seria razoável aumentar a qualidade dos produtos desconsiderando o aumento dos custos, sob pena de entregar um produto com excelente qualidade, porém com um preço inaceitável pelo mercado consumidor (PAIVA, CARVALHO JÚNIOR; FENSTERSEIFER, 2002). O impacto das ferramentas e técnicas para melhoria do processo produtivo deve ser analisado conjuntamente frente a todos os requisitos dos clientes e os objetivos estratégicos da manufatura definidos pela organização.

Por fim, é necessário avaliar o retorno financeiro estimado pela aplicação de ferramentas de melhorias no sistema produtivo que atendem aos requisitos do mercado, prevendo os ganhos resultantes dos investimentos realizados. Dentre as várias alternativas disponíveis na literatura para estimar receitas futuras, os autores optaram pelo uso do CLV. Esta técnica é definida como o valor presente dos lucros futuros obtidos de um cliente durante o seu período de relacionamento com a empresa (GUPTA; ZEITHAML, 2006) e caracteriza-se como uma métrica capaz de mensurar e quantificar em termos monetários os resultados do relacionamento da empresa com os seus clientes. Para Jain e Sing (2002), modelos de CLV podem ser muito úteis para ajudar as empresas a direcionar as estratégias, assim como as decisões táticas.

\section{O modelo proposto}

O modelo conceitual agrega áreas distintas, mas igualmente importantes, que são: o mercado consumidor, o sistema de produção e a métrica para mensuração do impacto dos cenários de melhorias.

O modelo visa a suportar decisões estratégicas que regem investimentos em melhorias nos processos da empresa. Sob a estratégia da empresa, encontram-se o mercado consumidor com as suas necessidades, preferências e percepções, e o sistema de produção, que viabiliza a oferta dos bens e serviços ao mercado. O modelo estima o CLV dos consumidores, permitindo avaliar as variações no CLV dos clientes para diferentes cenários de melhorias no sistema de produção.

São elementos de entrada do modelo: as preferências estabelecidas pelos consumidores, a análise de capacidade do processo produtivo e a probabilidade de compra de cada consumidor usando a teoria probabilística de modelo logit de escolha. A saída do modelo são os CLV de cada consumidor.

A modelagem da relação entre as variáveis que medem os atributos que compõem a utilidade do bem ou do serviço analisado e as variáveis operacionais do processo produtivo permite avaliar o impacto de 
melhoria por meio do modelo proposto. Em suma, o modelo estima o CLV dos consumidores de um bem ou um serviço em função das suas preferências e percepções sobre o que a empresa entrega ao mercado em relação a essas preferências.

A Figura 2 apresenta o modelo conceitual contendo os elementos básicos da integração proposta entre requisitos de mercado, sistemas de produção e métrica CLV.

O modelo proposto parte de uma Equação que permite o cálculo do CLV dos clientes. A partir do modelo CLV, as demais relações apresentadas são agregadas, visando a estimar o impacto de investimentos em melhoria do processo produtivo no CLV dos clientes. A Equação básica de CLV utilizada no modelo foi proposta por Berger e Nasr (1998), conforme apresentado na Equação 1.

$$
C L V=\sum_{i=1}^{n} \frac{\left(R_{i}-C_{i}\right)}{(1+d)^{n}}
$$

O modelo básico apresentado por esses autores considera as receitas geradas pelo cliente em cada período e os custos diretos com marketing, como mala direta, promoções, amostras grátis, entre outros (BERGER; NASR, 1998). No modelo proposto neste artigo, o CLV é um elo interligado com o processo produtivo. Portanto, os custos operacionais e variáveis como, por exemplo, mão-de-obra e material gasto para atender as ordens de produção, serão levados em conta na variável custo.

$\mathrm{Na}$ forma ora apresentada, o modelo é proposto para empresas que operam em mercados de produtos e serviços adequados para cada cliente sob a forma de um projeto. Apenas os custos que podem ser alocados diretamente no projeto (material e mão-de-obra produtiva) serão considerados.

Na Equação 1, o parâmetro $d$ representa a taxa de desconto. Normalmente, utiliza-se a taxa de juros básica da economia ou a taxa de desconto utilizada regularmente pela empresa em função do seu custo de capital. O parâmetro $n$ significa o número de períodos considerados (horizonte) na análise. O parâmetro $R$ representa a projeção da receita gerada pelo cliente no período $i$.

O CLV requer a modelagem do fluxo de receitas futuras. Como a variável receita futura depende diretamente da demanda, normalmente há duas incertezas na estimação da demanda: 'qual é o tamanho da demanda primária' e 'qual será o market share, auferido com base na competitividade entre concorrentes diretos e indiretos por essa demanda'. Para o modelo ora apresentado, assume-se que há informação suficiente sobre a demanda primária. Esse é o caso, por exemplo, de empresas de manutenção técnica de equipamentos de grande porte. Nesse contexto, com base nos requisitos da legislação e em informações de confiabilidade e durabilidade dos equipamentos (hazardmodels), pode-se estimar a demanda primária ao longo do tempo. Para o caso de mercados menos regulados, pode-se reduzir a incerteza sobre a demanda primária com base em modelos de séries temporais que levem em conta elementos macroeconômicos de avaliação da economia e fatores de renovação e ciclo de vida de produtos. Naturalmente, não se pode eliminar a incerteza sobre o market share, mas se pode reduzi-la utilizando modelos de escolha discreta, baseados na teoria da

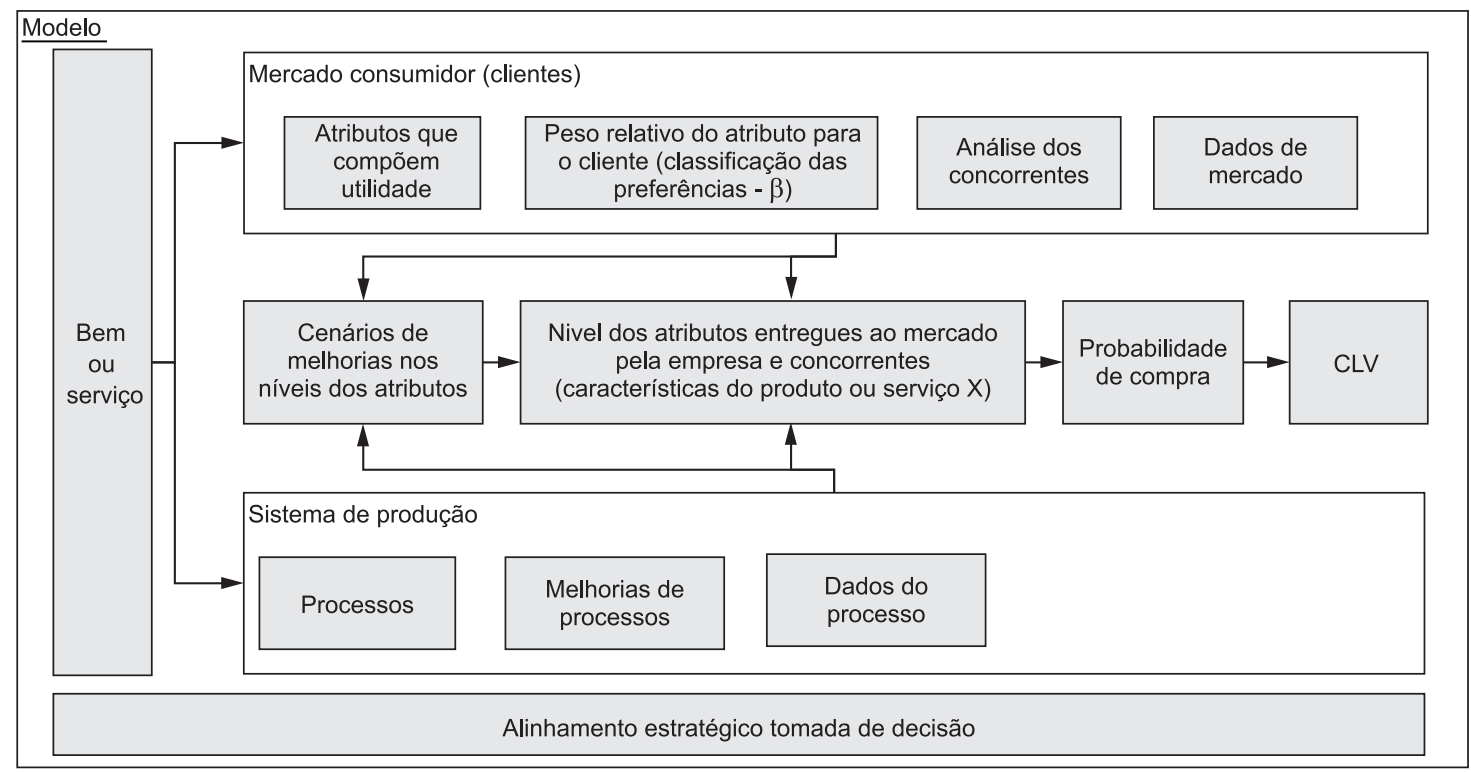

Figura 2. Modelo conceitual proposto. 
utilidade esperada (NEUMANN; MORGENSTERN, 1947; TRAIN, 2003).

Os custos são representados por $\mathrm{C}_{\mathrm{i}}$ e resultam das despesas com matéria-prima adquirida diretamente para cada projeto e com mão-de-obra produtiva alocada diretamente no desenvolvimento das atividades do projeto. Conforme o contexto estabelecido, a mão-de-obra é alocada apenas durante o projeto, sendo liberada após seu término. Para fins de simplificação da discussão, assume-se por ora que a mão-de-obra não seja limitada ou subordinada à competitividade de mercado, não havendo, portanto, custos adicionais de manutenção ou fidelização da mesma. Assim, a variável $\mathrm{C}_{\mathrm{i}}$ é composta por dois componentes: $\mathrm{C}_{1 \mathrm{i}}$ e $\mathrm{C}_{2 \mathrm{i}}$. A eliminação das perdas, ineficiências e desperdícios de mão-de-obra e material implicam reduções nos custos; o modelo será capaz de absorver essas flutuações mediante fatores, $\mathrm{k}_{1 \mathrm{i}}$ e $\mathrm{k}_{2 \mathrm{i}}$, que representam a razão entre a quantidade de material e mão-de-obra normalmente utilizados em cada projeto pela quantidade demandada pelo processo após as melhorias obtidas nas simulações do sistema produtivo.

O modelo proposto inclui outra característica flexível para representar multas contratuais, frequentes em projetos de grande porte. A multa $\left(\mathrm{C}_{3 \mathrm{i}}\right)$ causa impacto nos custos do projeto. Cada projeto entregue fora do prazo de entrega estipulado em contrato acarreta em uma multa, definida pelo produto entre os dias em atraso pelo valor diário da multa. Esse termo é acrescido ao modelo como uma variável que realiza descontos sobre os resultados das receitas propiciados pelos projetos em cada período. Por isso, um fator $k_{3 \mathrm{i}}$ que atenue o impacto da multa no resultado do CLV é incluído no modelo. Portanto, a variável custo em cada período $\left(\mathrm{C}_{\mathrm{i}}\right)$ é definida conforme a Equação 2.

$$
C_{i}=k_{1 i} C_{1 i}+k_{2 i} C_{2 i}+k_{3 i} C_{3 i}
$$

Adicionalmente, o modelo inclui um modelo de escolha discreta, para estimar o market share da empresa por meio das características dos produtos e serviços oferecidos, e dos requisitos do mercado. Modelos de escolha discreta são obtidos a partir de uma consideração sobre a forma da distribuição conjunta dos componentes aleatórios na função utilidade Von Neumann-Morgenstern. (NEUMANN; MORGENSTERN, 1947; TRAIN, 2003). Dentre os modelos de escolhas discreta disponíveis, utilizou-se o modelo logit.

O modelo logit agregado ao modelo CLV estima em cada período a probabilidade de compra do cliente, considerando as informações provenientes do mercado (preferências dos clientes e características da oferta dos concorrentes) e do sistema produtivo da empresa. Este fator será denominado $r_{\mathrm{i}}$ e indica a probabilidade de compras do cliente em cada período. $\mathrm{O}$ modelo logit (Equação 3) estima a probabilidade através da relação entre a exponencial da utilidade da empresa (potencial de que o cliente compre os produtos ou serviços da empresa) e a soma das utilidades das empresas concorrentes.

$$
r_{i}=\frac{e^{U_{E M P, i}}}{e^{U_{E M P, i}}+\sum_{j=1}^{m} e^{U_{C_{j}, i}}}
$$

em que:

$U_{E M P, i}:$ utilidade da empresa analisada $\left(U_{E M P} \in[0 ; 1]\right)$;

$U_{c j, i}:$ utilidade do concorrente $j\left(U_{c j} \in[0 ; 1]\right.$,

$0 \leq \mathrm{j} \leq m)$; $\mathrm{e}$

$m$ : número de concorrentes.

Se o produto ou o serviço oferecido não satisfaz os requisitos mais importantes para o cliente, então as probabilidades de compra são baixas. Quando são realizadas melhorias nos fatores-chaves, as probabilidades de compras em períodos futuros tenderão a aumentar.

Agregando as Equações 2 e 3, na Equação 1, tem-se omodelo proposto, conforme apresentado na Equação 4:

$$
C L V=\sum_{i=1}^{n} r_{i} \frac{\left(R_{i}-\left(k_{1 i} C_{1 i}+k_{2 i} C_{2 i}+k_{3 i} C_{3 i}\right)\right)}{(1+d)^{n}}
$$

A regra da função utilidade assume que a preferência de um indivíduo por uma alternativa é mensurável (LANCASTER, 1966). Portanto, para a determinação da utilidade (U), são necessários dois dados provenientes do mercado: o grau de importância atribuído pelo cliente a cada requisito e as características impostas pelo processo produtivo aos produtos e serviços nos fatores indicados pelos clientes. A função utilidade é definida pela relação linear entre os vetores contendo os pesos dos requisitos e as características dos produtos e serviços, conforme a Equação 5:

$$
U_{j}=\beta_{k} \cdot X_{j k}
$$

em que:

$U_{j}$ : Utilidade do j-ésimo indivíduo (empresa/ concorrentes) $\left(U_{j} \in[0 ; 1]\right)$;

$\beta_{k}$ : É um vetor contendo os pesos relativos atribuídos pelos clientes aos k requisitos na composição da função utilidade;

$X_{j k}$ : É um vetor contendo os atributos dos produtos e serviços nos $\mathrm{k}$ requisitos para o indivíduo $\mathrm{j}$.

Diferentes requisitos podem ser agregados ao modelo, desde que corretamente identificados no mercado. Para isso, basta incluir esses novos fatores ao vetor $\beta$ e identificar o link com o sistema produtivo e os possíveis impactos nas variações das variáveis custos e receitas. Justamente é nessa fase que as ferramentas 
e informações do marketing são necessárias para assegurar robustez ao modelo.

Agregando as Equações 3 e 5, obtém-se a Equação 6, que apresenta a probabilidade de compras pelo cliente em cada período.

$$
r_{i}=\frac{e^{\left(\beta_{k} \cdot X_{E M P k}\right)}}{e^{\left(\beta_{k} \cdot X_{E M P k}\right)}+\sum_{j=1}^{m} e^{\left(\beta_{k} \cdot X_{C_{j} k}\right)}}
$$

Adicionando a Equação 4 na 6, obtém-se a Equação Geral do modelo proposto, conforme Equação 7:

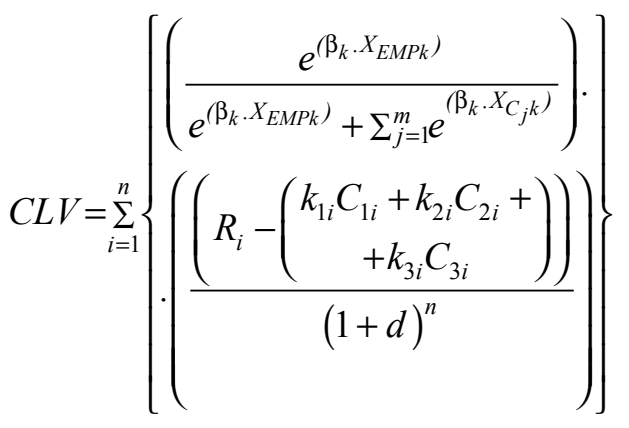

A seguir, é apresentada uma breve discussão sobre técnicas para a estimação dos termos necessários para a aplicação do modelo.

\section{Considerações sobre a mensuração de variáveis do modelo}

O primeiro passo para a aplicação do modelo é obter os dados do mercado, que são os requisitos importantes para os clientes para um dado produto ou serviço, o grau de importância de cada requisito e as características do processo produtivo que impactam nos atributos importantes. O segundo passo consiste em mensurar as variáveis, como as características do processo produtivo dos concorrentes e as receitas e despesas futuras. As informações do mercado podem ser obtidas por meio de ferramentas qualitativas e quantitativas, como entrevistas, grupo de foco, entre outras (MALHOTRA, 2006). Os dados referentes ao processo produtivo são obtidos por meio de simulações desse processo.

A identificação dos requisitos relevantes para os clientes ocorre por meiode métodos qualitativos, como entrevistas com especialistas do setor e grupos focados com os clientes, que auxiliam na identificação de quais elementos e fatores impulsionam a decisão de contratação do serviço ou compra do produto pelo cliente. Os resultados dessa etapa servem de entrada para o segundo conjunto de técnicas quantitativas que visam a estimar o grau de importância de cada requisito para o cliente e o desempenho da empresa frente à concorrência em cada requisito. Essas técnicas quantificam as preferências dos clientes e, para isso, são utilizadas técnicas tradicionais de marketing, como a Análise Conjunta, apresentadas por Urban e Hauser (1993), Malhotra (2006) e Nunnally e Bernstein (1994). Essas ferramentas e técnicas devem identificar a interação entre os diversos fatorespara, assim, compreender e considerar os trade offs anteriormente discutidos.

Dentre as técnicas existentes para identificar a interação entre os diferentes fatores, destacam-se o Analytic Hierarchy Process (AHP) e a Análise Conjunta (Conjoint Analysis).

O fundamento do método AHP está baseado na decomposição e na síntese das relações entre os critérios (requisitos do cliente) até encontrar uma priorização dos seus indicadores, aproximando-se de uma melhor resposta de medição única de desempenho (SAATY, 1991). O método AHP apresenta fragilidades no sentido de não fornecer garantias de confiabilidade, apesar de permitir a estimação da consistência das respostas obtidas. A Análise Conjunta é uma técnica estatística que permite determinar o peso de diferentes características que compõem um determinado produto ou serviço. O objetivo é determinar a influência de cada requisito no processo de escolha e tomada de decisão dos clientes (GREEN; CARROLL; GOLDBERG, 1981). Para identificar se todos os avaliadores (clientes) estão avaliando na mesma direção, o método Proportional Reduction in Loss (PRL) permite o cálculo da confiabilidade das medições e a assimetria nas avaliações dos respondentes (RUST; COOIL, 1994).

A tradução dos requisitos do mercado para o sistema produtivo é efetuada pelo método QFD. As intensidades das melhorias nas características do processo produtivo, por sua vez, são identificadas por meio de simulação computacional. Nesse caso, o processo produtivo é reproduzido em um modelo interativo capaz de informar os dados necessários aos indicadores atrelados aos requisitos do cliente. Com esse modelo computacional, algumas ferramentas e técnicas de melhorias no processo podem ser testadas gerando os dados necessários para o estudo de viabilidade de investimentos em melhoria a partir do modelo proposto na seção anterior. Essa etapa requer um conhecimento detalhado dos elementos relevantes do processo analisado, desde os fluxos da produção até as informações de tempos de operação, quantidades de mão-de-obraematerial envolvidos, entre outros.

O uso de modelos logit pressupõe que a oferta/ disponibilidade produtiva é igual para todos os players, ou seja, o cliente pode escolher livremente entre todos os concorrentes sem restrição de não ser atendido por falta de capacidade produtiva. Como as informações dos concorrentes são indispensáveis para o sucesso do modelo e devido à dificuldade em obter tais dados diretamente dos mesmos, utilizam-se informações dos próprios clientes para estimar o desempenho dos concorrentes. Para isso, pressupõe-se que os clientes 
conheçam os produtos e serviços dos concorrentes tão bem quanto os da própria empresa analisada e sejam capazes de mensurar as características dos concorrentes em relação à empresa. Os dados dos concorrentes são então estimados a partir das características simuladas do processo produtivo da empresa.

A próxima seção descreve uma aplicação simplificada do modelo proposto em um contexto real. Essa aplicação busca compreender o comportamento do modelo quanto à resposta do mesmo para diferentes níveis de investimentos em melhorias.

\section{Aplicação do modelo}

O objeto de estudo é uma empresa prestadora de serviços de manutenção aeronáutica. Por lidar com equipamentos de alta tecnologia e transportar milhares de pessoas, essa é uma indústria altamente regulamenta por órgãos nacionais e internacionais, como, por exemplo, a Agência Nacional de Aviação Civil (ANAC) e o Federal Airspace Adminstration (FAA). Dentre o portfólio de serviços oferecidos pela empresa, o produto escolhido para a aplicação do modelo foi aquele que representa a maior importância para a empresa, no que tange ao seu faturamento. $\mathrm{O}$ serviço em questão pode ser compreendido como um projeto, no qual todas as atividades são programadas e planejadas em cada novo projeto, tendo muito bem definidas uma data de início e uma para conclusão do projeto; o resultado de cada serviço é único, caracterizando assim um projeto. Em cada projeto, as despesas com matéria-prima e mão-de-obra são alocadas diretamente às ordens de venda do cliente. Em caso de atraso no prazo contratual, aplica-se uma multa, prática natural nesse segmento de atividade. Cada projeto é composto de inúmeros subserviços que, por sua vez, contemplam diversas atividades. O planejamento para execução das atividades em cada projeto contém uma estimativa da quantidade de horas programadas e material necessário para execução, assim como a data prevista para o término. Contratempos e problemas não previstos na elaboração dos cronogramas são comuns, o que colabora para o atraso na conclusão dos projetos.

Para fins de ilustração do modelo proposto, um único cliente será considerado. No contexto analisado, o cliente selecionado representa mais de $30 \%$ do faturamento no produto/serviço analisado. Os requisitos demandados pelo cliente e os graus de importância a eles atribuídos foram identificados com base na experiência dos executivos da empresa. Os requisitos relevantes para o cliente no produto analisado foram: entrega confiável e custo. A importância no prazo de entrega baseia-se no fato de atraso na entrega acarretar grandes prejuízos ao cliente. Segundo Slack (2002), os fatores de competitividade atrelados aos requisitos do cliente são, respectivamente,confiabilidade, que depende de um processo confiável, e custos relacionados com alta produtividade. No caso analisado, assume-se que esses são os fatores ganhadores de novos pedidos. As características atribuídas aos requisitos apresentados anteriormente são: $\beta_{1}$ (atraso da última entrega), $\beta_{2}$ (média de atrasos nas entregas) e $\beta_{3}$ (preço do serviço). Essas variáveis encontram suporte nos trabalhos de Cobb (1995) e Mathaisel (2005), que defendem que os critérios e atributos vencedores de pedidos na indústria de manutenção aeronáutica são: alta qualidade, baixo custo e entrega rápida e confiável.

A partir das variáveis atribuídas aos requisitos de mercado, é possível estimar afunção utilidade, conforme apresentada na Equação 8, e o modelo logit, conforme a Equação 9. Para fins ilustrativos, esta aplicação assume que há apenas dois concorrentes. Para adicionar mais concorrentes, basta alterar o denominador da Equação 9, agregando a utilidade de cada novo concorrente.

$$
U_{j}=\beta_{1} X_{1 j}+\beta_{2} X_{2 j}+\beta_{3} X_{3 j}
$$

em que:

$X_{l j}$ : atributo confiabilidade (atraso do último projeto) para o indivíduo $j$;

$X_{2 j}$ : atributo confiabilidade (média dos atrasos nos projetos passados) para o indivíduo $j$;

$X_{3 j^{\prime}}$ atributo custo (custos para execução do projeto) para o indivíduo $j$;

$\beta_{1}$ : peso relativo atribuído pelo cliente ao requisito atraso do último projeto $X_{l j}$ na composição da função utilidade;

$\beta_{2}$ : Peso relativo atribuído pelo cliente ao requisito média dos atrasos nos projetos passados $X_{2 j}$ na composição da função utilidade;

$\beta_{3}$ : Peso relativo atribuído pelo cliente ao requisito preço do serviço $X_{3 j}$ na composição da função utilidade;

j: Indivíduo (empresa e concorrentes).

$$
r_{i}=\frac{e^{\beta_{1} X_{1, E M P, i}+\beta_{2} X_{2, E M P, i}+\beta_{3} X_{3, E M P, i}}}{\left(\begin{array}{c}
e^{\beta_{1} X_{1, E M P, i}+\beta_{2} X_{2, E M P, i}+\beta_{3} X_{3, E M P, i}}+ \\
e^{\beta_{1} X_{1, c 1, i}+\beta_{2} X_{2, c 1, i}+\beta_{3} X_{3, c 1, i}}+ \\
e^{\beta_{1} X_{1, c 2, i}+\beta_{2} X_{2, c 2, i}+\beta_{3} X_{3, c 2, i}}
\end{array}\right)}
$$

Os pesos relativos $\beta_{1}, \beta_{2}$ e $\beta_{3}$ utilizados nessa aplicação foram obtidos por meio de entrevistas com o cliente analisado. Esses dados estão resumidos na Tabela 1.

Todas as empresas do segmento aeronáutico são obrigadas a fazer manutenções periódicas e obrigatórias, pois a legislação vigente no setor exige que, em períodos de tempo pré-estabelecidos, diversas partes e componentes das aeronaves passem por processo de check e manutenção. Portanto, a 
demanda primária, nesse caso, é determinística, uma vez sabidas as condições da frota (ciclos de voo, modelo das aeronaves, etc.) da base de clientes.

Os dados gerais do produto analisado, como preço de venda; quantidade e valores de mão-de-obra e material orçados; tempo de execução acordado em contrato, e quantidade de unidades que devem receber manutenção em cada período, conforme determinado pela legislação vigente, são apresentados na Tabela 2. Esses são dados típicos, porém foram alterados para evitar exposição dos dados reais da empresa analisada.

As características do processo produtivo foram simuladas com o auxilio do software de simulação Micro Saint através de um modelo simplificadodo processo produtivo. Todas as distribuições de probabilidade utilizadas no modelo computacional do processo produtivo ilustrado na Figura 3 são normais, conforme resumido na Tabela 3. Não é sempre que, em um processo produtivo, os tempos de espera e de processamento distribuem-se normalmente. Nestes casos, deve-se identificar a distribuição mais apropriada para os dados, como, por exemplo, distribuição exponencial, weibull ou outras.

Naturalmente, o modelo segue premissas que limitam os resultados encontrados, mas viabilizam sua aplicação em um contexto real. Dentre as premissas para a presente aplicação, assume-se que a relação entre percentual de investimento e percentual de melhoria é inversamente proporcional. Ou seja, elevar em $20 \%$ o investimento em uma melhoria que reduza o tempo de processamento gera uma redução de $20 \%$ no tempo de processamento em questão. Uma análise mais detalhada da relação entre nível de investimento e impacto do investimento no processo produtivo é recomendada para estimar o efeito mais aderente entre estas variáveis. Essa premissa pode ser alterada a qualquer momento sem interferir no modelo proposto.
Os indicadores do processo produtivo apresentados na Tabela 4 foram normalizados em uma escala de -1 a 1 . Por exemplo, o fator $X_{1}$ indica que a empresa atrasou $24 \%$ além do prazo contratual de 35 dias, no último projeto entregue ao cliente analisado, e que o preço de venda do serviço pela empresa é o ponto central da escala.

Devido à dificuldade em conseguir as características do processo produtivo dos concorrentes, estimaram-se os valores dessas características com base no desempenho da empresa frente aos seus concorrentes em cada um dos requisitos obtidos na etapa de entrevistas com o cliente. A decisão de realizar negócios com a empresa é do cliente, portanto é justificável estimar as características do processo produtivo dos concorrentes a partir da percepção dos clientes. A Tabela 5 apresenta valores arbitrados, com base nas percepções do cliente, sobre o desempenho da empresa frente a seus dois concorrentes em cada um dos três requisitos. Nota-se que a empresa possui desempenho inferior nos indicadores $\mathrm{X}_{1}$ e $\mathrm{X}_{2}$ para ambos os concorrentes, embora seu preço de venda $\left(\mathrm{X}_{3}\right)$ seja inferior ao dos concorrentes.

A Tabela 6 resume as características do processo produtivo dos concorrentes normalizadas, estimadas a partir das informações contidas na Tabela 5.

Com as informações apresentadas, é possível obter o CLV do cliente para um horizonte de tempo de cinco anos. O modelo apresenta um valor de CLV estimado de $\mathrm{R} \$ 1.229 .197,00$, ou seja, o retorno atualizado para o presente esperado pela empresa para esse cliente nos próximos cinco anos. Como o desempenho da empresa não é satisfatório nos principais requisitos do cliente, faz-se necessário promover melhorias no processo produtivo de forma a ajustar os fatores $X_{1}$, $\mathrm{X}_{2}$ e $\mathrm{X}_{3}$. Assim, diferentes melhorias no processo foram propostas e simuladas em interações variando entre 0 e $100 \%$ no efeito da melhoria. O conjunto de

Tabela 1. Requisitos do mercado para o cliente analisado.

\begin{tabular}{|c|c|c|c|}
\hline Requisitos do cliente & Entrega confi & o de entrega) & Baixo preço \\
\hline Objetivo de desempenho & \multicolumn{2}{|c|}{ Confiabilidade } & Custos \\
\hline Indicador & Último atraso $\left(\beta_{1}\right)$ & Média atrasos $\left(\beta_{2}\right)$ & Preço de venda $\left(\beta_{3}\right)$ \\
\hline Relevância & 4,5 & 3,0 & 4,0 \\
\hline
\end{tabular}

Tabela 2. Dados gerais do serviço contratado pelo cliente analisado.

$\begin{array}{lc}\text { Prazo execução do projeto (contratual) [dias] } & 35 \\ \text { Demanda primária do cliente por período i [unidades] } & 10 \\ \text { Preço de venda do Projeto [reais] } & \mathrm{R} \$ 1.000 .000,00 \\ \text { Unidades de material orçadas [unidades] } & 6200 \\ \text { Custo unitário com material [real/unidade] } & \mathrm{R} \$ 36,76 \\ \text { Unidades de mão-de-obra orçada [unidades] } & 10000 \\ \text { Custo unitário com mão-de-obra [real/unidade] } & \mathrm{R} \$ 46,30 \\ \text { Multa contratual [real/dia] } & \mathrm{R} \$ 5.000,00\end{array}$


Tabela 3. Distribuições utilizadas na simulação.

\begin{tabular}{lllll}
\hline \multicolumn{1}{c}{ Processo } & \multicolumn{4}{c}{ Distribuição } \\
\hline Espera material & $\mathrm{E} 1 \sim \mathrm{N}(48,24)$ & $\mathrm{E} 2 \sim \mathrm{N}(24,12)$ & $\mathrm{E} 3 \sim \mathrm{N}(24,12)$ & $\mathrm{E} 4 \sim \mathrm{N}(24,12)$ \\
Espera ferramentas & $\mathrm{F} 1 \sim \mathrm{N}(12,6)$ & $\mathrm{F} 2 \sim \mathrm{N}(12,6)$ & $\mathrm{F} 3 \sim \mathrm{N}(12,6)$ & $\mathrm{F} 4 \sim \mathrm{N}(12,6)$ \\
Processamento & $\mathrm{P} 1 \sim \mathrm{N}(120,20)$ & $\mathrm{P} 2 \sim \mathrm{N}(240,60)$ & $\mathrm{P} 3 \sim \mathrm{N}(310,40)$ & $\mathrm{P} 4 \sim \mathrm{N}(120,20)$ \\
Deslocamentos & $\mathrm{D} 1 \sim \mathrm{N}(12,6)$ & $\mathrm{D} 2 \sim \mathrm{N}(0,0)$ & $\mathrm{D} 3 \sim \mathrm{N}(12,6)$ & \\
\hline
\end{tabular}

Tabela 4. Características do processo produtivo da empresa.

\begin{tabular}{lccc}
\hline \multicolumn{1}{c}{ Característica } & Último atraso (X1 EMP) & Média atrasos (X2 EMP) & Preço do serviço (X3 EMP) \\
\hline Situação Inicial & 0,24 & 0,31 & 0 \\
\hline
\end{tabular}

Tabela 5. Desempenho da empresa frente aos concorrentes.

\begin{tabular}{cccc}
\hline Indicador & Último atraso $\left(\mathbf{X}_{\mathbf{1}}\right)$ & Média atrasos $\left(\mathbf{X}_{\mathbf{2}}\right)$ & Preço do serviço $\left(\mathbf{X}_{3}\right)$ \\
\hline Desempenho concorrente 1 & $30 \%$ & $25 \%$ & $-10 \%$ \\
Desempenho concorrente 2 & $35 \%$ & $35 \%$ & $-15 \%$ \\
\hline
\end{tabular}

Tabela 6. Características do processo produtivo dos concorrentes.

\begin{tabular}{rccc}
\hline Indicador & Último atraso $\left(\mathbf{X}_{\mathbf{1}} \mathbf{C} \mathbf{j}\right)$ & Média atrasos $\left(\mathbf{X}_{\mathbf{2}} \mathbf{C} \mathbf{j}\right)$ & Preço do serviço $\left(\mathbf{X}_{\mathbf{3}} \mathbf{C} \mathbf{j}\right)$ \\
\hline Concorrente 1 & 0,17 & 0,23 & 0,10 \\
Concorrente 2 & 0,15 & 0,20 & 0,15 \\
\hline
\end{tabular}

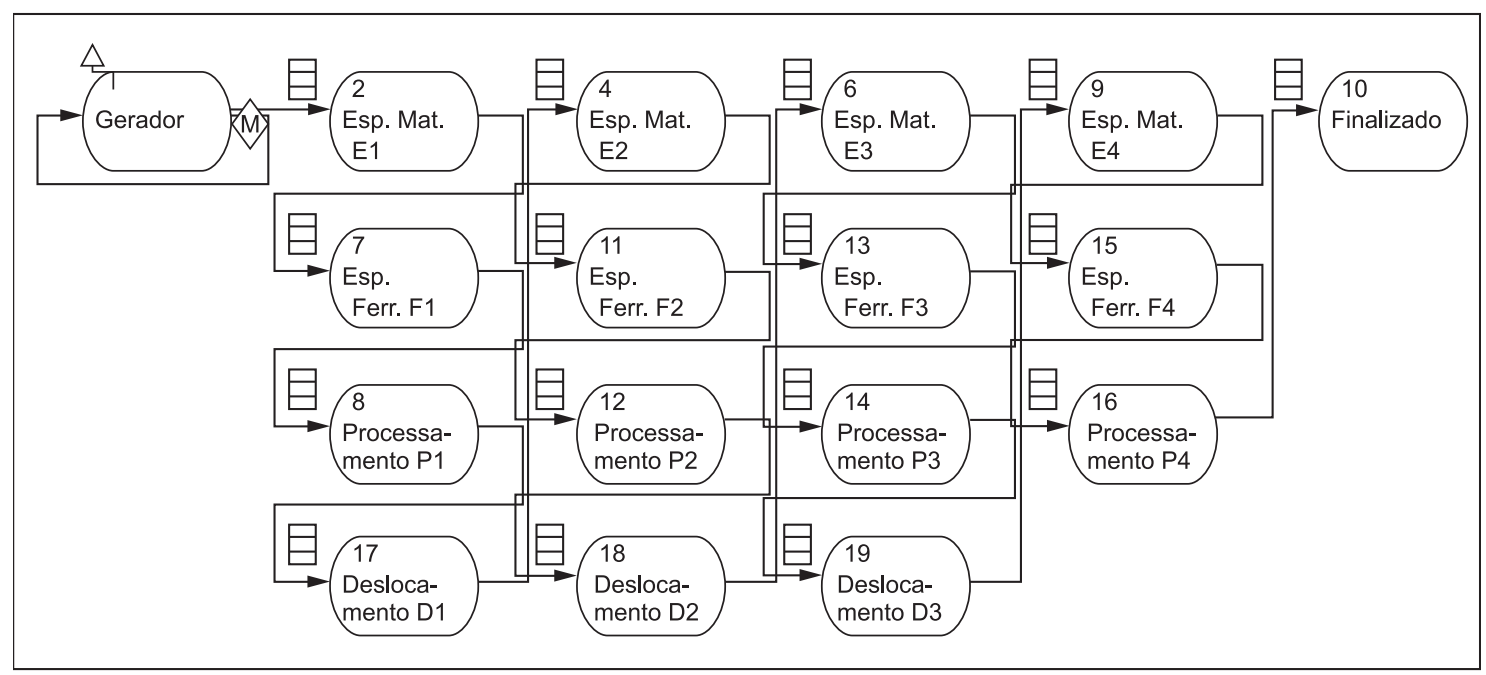

Figura 3. Simulação simplificada do processo produtivo.

melhorias e indicadores propostos nesse caso parte dos conceitos de Lean Manufacturing. Mathaisel (2005) apresenta um resumo de ferramentas Lean aplicadas na indústria analisada e respectivas contribuições ao processo. As melhorias no processo produtivo e os indicadores sugeridos e simulados estão listados na Tabela 7.

A partir das melhorias propostas (M1, M2, M3 e M4), 40 cenários distintos foram construídos por meio da variação da eficiência de cada melhoria no processo de 0 a $100 \%$, em passos de $10 \%$. Em cada cenário simulado, os demais parâmetros foram mantidos inalterados. A redução na variabilidade implica redução dos desperdícios, resultando em menores quantidades de matéria-prima e mão-deobra. A partir das simulações do processo em cada uma das quatro melhorias propostas para o range de ajuste citado, calcularam-se os novos valores de $\mathrm{X} 1$ e X2. A partir dos novos valores de X1 e X2, calculou-se novamente o CLV do cliente para cada 
cenário de melhoria simulado, gerando os resultados apresentados na Figura 4.

$\mathrm{O}$ eixo X da Figura 4 apresenta o percentual de melhorias em M1, M2, M3 e M4. Ao investir 200 mil reais na melhoria M1, é possível obter $100 \%$ de redução nos tempos de deslocamentos no processo produtivo. Analogamente, ao investir 300 mil reais em M2, o tempo de setup é reduzido em 100\%; 500 mil reais em M3, os tempos de espera podem ser reduzidos em $100 \%$, e quatro milhões de reais em M4, as variabilidades do processo são reduzidas em $100 \%$. A atenuação dos problemas no processo simulado é proporcional ao volume de investimento; por exemplo, investir 100 mil reais em M1 resulta em uma redução dos tempos em 50\%, conforme premissa discutida anteriormente. $\mathrm{O}$ eixo $\mathrm{Y}$ da Figura 4 apresenta o delta CLV do cliente (isto é, a diferença entre o CLV do cliente antes e depois da melhoria) dividido pelo total de recursos investidos em melhorias em cada cenário simulado.

A análise da Figura 4 aponta a melhoria M3 em um baixo nível de investimento (10\%) como a situação que apresenta o melhor retorno sobre o investimento em melhorias.

Como as variáveis $\mathrm{X}_{1}$ e $\mathrm{X}_{2}$ são dependentes do tempo de entrega do projeto, pode ser observado na simulação do processo que, ao reduzir em $10 \%$ o tempo de espera por material, o atraso do projeto foi reduzido em aproximadamente $47 \%$ para um investimento da ordem de 50 mil reais. Diferentemente, o menor tempo de entrega encontrado em todos os

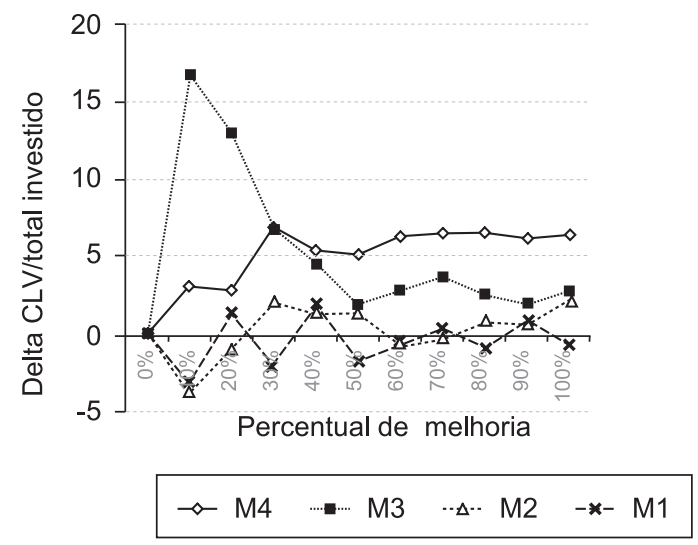

Figura 4. Comportamento do processo frente às melhorias.
40 cenários simulados foi $72 \%$ para um investimento de $100 \%$ em M3, totalizando 500 mil reais.

$\mathrm{O}$ evento espera por material representa boa parte do atraso do projeto e as melhorias realizadas nesse ponto provocam elevado índice de redução nos tempos de entrega dos projetos. Essa redução enfrenta um ponto de saturação, ponto esse em que filas começam a ser formadas nas atividades mais lentas do processo, que é o processamento A3 (Figura 3). A partir desse ponto, reduzir o tempo de espera por material deixa de ser tão eficiente na redução do tempo de entrega mesmo que cresça o volume de investimentos.

A melhoria M4 apresenta comportamento similar ao da melhoria M3 no que tange ao encontro do ponto de saturação do sistema. Após o índice de 30\% de redução na variabilidade do processo, a relação delta CLV e investimento torna-se praticamente estável pelo fato de que o aumento do volume de investimento é proporcional ao volume de redução das despesas nos custos do projeto, ocasionados pela diminuição na quantidade de mão-de-obra e desperdícios de material. Esse resultado evidencia que não há o porquê de investir elevado volume de recursos em reduzir a variabilidade mais que $30 \%$ em relação aos níveis correntes.

Nas condições analisadas, as demais melhorias propostas, M1 e M2, não apresentam resultados significativos pelo fato de estas impactarem pouco no tempo total de entrega dos projetos e seus custos de implantações serem elevados. Os melhores resultados nos tempos de entrega do projeto na melhoria M1 foi uma redução de $10 \%$ no tempo de deslocamento com um de investimento de 80 mil reais e, para a melhoria M2, uma redução de $40 \%$ no tempo de setup de ferramentas com um investimento de 300 mil reais.

A Figura 5 ilustra os valores absolutos do valor do CLV do cliente para os cenários de melhorias simulados para as quatro melhorias propostas. Nessa figura, é possível visualizar o efeito na redução dos custos provocados pela melhoria $\mathrm{M} 4$. O crescimento no valor do CLV, nessa situação, supera os investimentos de implantação.

A análise da Figura 5 permite confirmar as análises obtidas na Figura 4. Percebe-se que a melhoria M3 apresenta o maior retorno financeiro no valor do cliente para um baixo percentual de investimento. Em termos absolutos, a melhoria M4 gera o maior

Tabela 7. Melhorias no processo produtivo.

\begin{tabular}{cc}
\hline Melhorias & Descrição da melhoria \\
\hline M1 & Alterar layout para reduzir deslocamentos (reduzir deslocamentos entre 0 e $100 \%)$ \\
M2 & Setup de Ferramentas (reduzir espera por ferramentas entre 0 e 100\%) \\
M3 & Ajustar processo logístico material (reduzir entre 0 e 100\% o tempo espera material) \\
M4 & Reduzir a variabilidade das atividades (reduzir todas as variabilidades entre 0 e $100 \%)$ \\
\hline
\end{tabular}


incremento no CLV do cliente, mas requer elevados volumes de investimentos.

O modelo proposto possibilitou à empresa conhecer e comparar quantitativa e objetivamente o resultado de diferentes níveis de investimento em diferentes ferramentas de melhoria do sistema produtivo.

$\mathrm{O}$ caso apresentado ao longo deste artigo serve como exemplo para ilustrar a aplicação do modelo proposto. Outras aplicações e extensões podem ser realizadas no futuro, como avaliar o impacto da combinação desses elementos de melhoria (por exemplo, comparando M1+M2 com M3+M2).

\section{Análise crítica dos resultados e considerações finais}

O modelo proposto neste artigo e aplicado no caso apresentado anteriormente carece de aplicações mais robustas para que afirmações consistentes sobre o modelo sejam formuladas. No entanto, o modelo proposto apresentou resultados que efetivamente podem auxiliar a tomada de decisão sobre investimento em melhorias em sistemas produtivos.

Esta pesquisa não objetiva validar o modelo proposto, sendo o mesmo simplesmente aplicado ao caso descrito. Essa aplicação possibilitou a geração de resultados práticos úteis a esta situação e a análise de viabilidade de aplicação a partir da experiência deste caso. Entretanto, esses resultados não são conclusivos e não há garantias de que as análises sobre viabilidade são validas para futuras aplicações.

A modelagem do processo produtivo apresenta simplificações, dentre as quais: a relação linear entre o nível de investimento e o percentual de melhoria, as distribuições normais e a omissão de avaliação do efeito de cada melhoria sugerida em cada atividade apresentada, mas novamente tais simplificações podem ser alteradas sem impacto no modelo proposto. A simulação desenhada não previu variações no preço de venda do serviço $\left(\mathrm{X}_{3}\right)$ e, portanto, essa variável foi

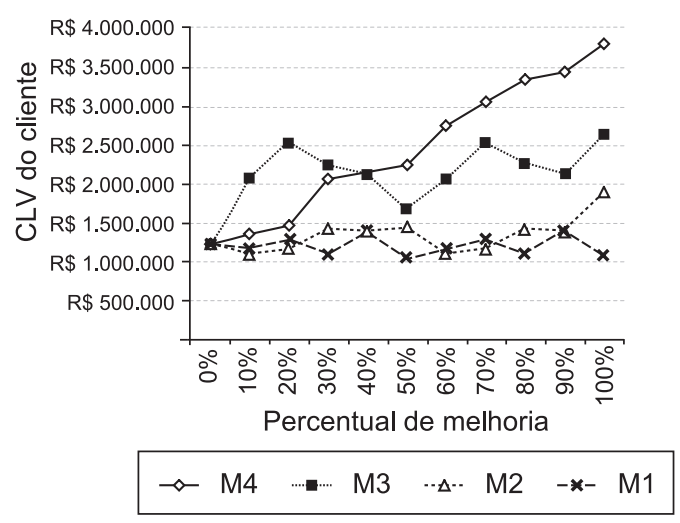

Figura 5. Valores absolutos CLV do cliente. mantida constante. A sua variação poderia apresentar resultados distintos dos apresentados.

As discussões apresentadas em cada item do método devem ser exploradas em maior profundidade, principalmente as que envolvem os conceitos, técnicas e ferramentas de produção defendida por diferentes correntes; dentre estas, é possível citar TOC, STP, Lean-Sigma, etc. O modelo exige um grande conhecimento prévio nas ferramentas e técnicas apresentadas.

A aplicação do modelo em um caso real demonstrou a necessidade de um método estruturado para a aplicação do modelo em contextos reais, visto que ele demanda uma grande quantidade de dados oriundos de diferentes fontes. A aplicação do modelo torna-se trabalhosa no momento em que elevadas quantidades de dados de distintas fontes devem ser coletadas, estimadas e observadas, o que limita o campo de aplicação do modelo a ambientes organizados e em que não ocorram grandes variações nas preferências dos consumidores em curto espaço de tempo.

Dada a dificuldade de aplicação do modelo, o mesmo torna-se atrativo quando a seguinte pergunta necessita ser respondida: "Por que os resultados gerados com o modelo não poderiam ser obtidos de um simples modelo de simulação de processo?". A resposta a essa questão é: primeiramente, o simples uso de simulação não disponibiliza dados sobre expectativas de elevação do ganho em função de melhorias, conforme realizado pelo modelo proposto; além disso, como saber se o gargalo ou CCR que limita o processo é significante aos consumidores, a ponto de que determinada melhoria neste gargalo ou CCR elevará o ganho global da empresa? O modelo proposto responde a essa questão, ao passo que o simples modelo de simulação do processo produtivo, não.

Como observação final, percebeu-se, pela aplicação realizada, que o modelo viabilizou uma análise das melhorias de processo em relação às preferências do mercado; porém, novas aplicações são recomendadas para propiciar uma validação mais consistente dos resultados observados e da estrutura do modelo em si.

\section{Conclusões}

Esta pesquisa desenvolveu um modelo para avaliar cenários de melhorias no sistema de produção a partir das preferências declaradas pelos consumidores. $\mathrm{O}$ modelo baseou-se na métrica CLV e em um modelo logit de escolha discreta. O CLV quantifica o ganho gerado por cada cliente de uma empresa e o modelo logit quantifica a relação entre as preferências declaradas pelos consumidores e as características percebidas pelos consumidores nos bens e serviços produzidos por uma empresa.

Apesar da variedade de conceitos e técnicas disponíveis na literatura abordando o tema melhorias no 
sistema de produção, pouco foi encontrado tratando o tema à luz das preferências declaradas pelos consumidores. Normalmente, as avaliações do processo produtivo focam no desempenho de indicadores e variáveis endógenas ao processo, como tempo de atravessamento e tempo de espera. Nestes casos, a justificativa de investimento realiza-se a partir dessas variáveis, deixando de considerar relações causais com variáveis exógenas ao sistema de produção.

A justificativa de investimentos no sistema produtivo pode ser reconhecida a partir de um projeto de simulação, que pressupõe tão somente variabilidades nas operações e atividades que compõem o processo ao gerar as respostasque servem de base para a tomada de decisão. Os cenários de melhorias são construídos para avaliar os efeitos dessas variabilidades de modo a identificar a opção que proporciona melhor resultado. A premissa básica da abordagem de simulação é que o problema a ser corrigido é conhecido a priori e o mesmo é obtido a partir de análises no sistema de produção, assumindo que o sistema produtivo é um sistema fechado.

O modelo proposto analisa os problemas a serem corrigidos no sistema de produção de um dado bem ou serviço, a partir das preferências declaradas pelos consumidores. Essa é a primeira diferença entre o método proposto e o método de simulação tradicional proposto por autores como Law e Kelton (1991). Diferentemente das técnicas tradicionais de simulação computacional de processos, esta pesquisa foca não somente na variabilidade imposta pelo processo produtivo, mas também na variabilidade nas preferências dos consumidores. O método proposto pressupõe que a preferência dos consumidores em cada atributo obtido a partir de uma amostra de consumidores assume uma distribuição de probabilidade. Possibilita-se, assim, que o grau de preferências de cada consumidor da população em cada atributo seja estimado.

Em termos práticos, a abordagem proposta agrega um novo nível de informação para auxiliar a tomada de decisão. Nos moldes usuais, avaliam-se os resultados das simulações sobre uma determinada variável, como, porexemplo, tempo de espera para decidir o que fazer para ajustar o processo produtivo. Diversamente, o método proposto identifica junto aos consumidores se o tempo de espera é relevante na utilidade gerada pelo produto ou serviço; caso seja relevante, é possível verificar o peso de importância do atributo tempo de espera para então gerar melhorias no sistema de produção para auxiliar na tomada de decisão.

Avaliar um único atributo, como o tempo de espera, não justifica a aplicação do modelo proposto. Entretanto, avaliar um único atributo tipicamente não condiz com a realidade do processo decisório, pois as escolhas dos consumidores são realizadas a partir de um conjunto de atributos. Considerando-se essa afirmação, a aplicação do método proposto se justifica, pois as preferências dos consumidores sobre esse conjunto de atributos são quantificadas e utilizadas pelomodelo.

A integração entre as áreas marketing e produção foi realizada de forma natural pelo modelo. A produção, fornecendo subsídio para as métricas e técnicas de marketing, com o objetivo em comum de estabelecer uma estratégia de investimentos baseada no sistema produtivo priorizando as necessidades dos clientes. Com isso, foi possível desenvolver uma abordagem integrada descrita por Hess e Lucas (2004) como "to do the right thing right".

Como pontos de extensão futura, o modelo necessita ser validado em um processo produtivo completo e não simplificado como o apresentado nesse artigo; é necessário também ser testado em diferentes cenários, sendo a interação entre os mesmos via planejamento de experimentos fatoriais. O modelo ainda possui margem para receber um fator que permita a avaliação do atraso entre a tomada de decisão na implantação da melhoria e a efetivação nos resultados financeiros. Por fim, pode ser estabelecido um método para aplicação sistemática do modelo em diferentes ambientes.

\section{Agradecimentos}

Os autores agradecem ao colega Guilherme Liberali Neto pelas valiosas contribuições e à UNISINOS pelo provimento dos recursos para a realização do presente trabalho.

\section{Referências}

ANTUNES JUNIOR, J. A. V. et al. Sistemas de Produção: Conceitos e Práticas para Projetos e Gestão da Produção Enxuta. Porto Alegre: Artmed, 2008. 326 p.

BERGER, P. D.; NASR, N. I. Customer lifetime value: Marketing models and applications. Journal of Interactive Marketing, v. 12, n. 1 p. 18-30, 1998. http://dx.doi.org/10.1002/(SICI)15206653(199824)12:1\%3C17::AID-DIR3\%3E3.0.CO;2-K

CHENG, L. C.; MELLO FILHO, L. D. R. QFD: Desdobramento da Função Qualidade na Gestão de Desenvolvimento de Produtos. São Paulo: Editora Blucher, 2007. 539 p.

COBB, R. Modeling aircraft repair turn time: Simulation supports maintenance marketing efforts. Journal of Air Transport Management, v. 2, n. 1, p. 25-32, 1995. http://dx.doi.org/10.1016/0969-6997(95)00024-6

FRANCO, A. L. Estratégia de Operações como Arma Competitiva em Serviços de Engenharia de Avaliações: Um Estudo de Caso. 2001. 134 f. Dissertação (Mestrado em Administração)-Universidade do Rio Grande do Sul, Porto Alegre, 2001.

GODINHO FILHO, M.; FERNANDES, F. C. F. Paradigmas Estratégicos de Gestão da Manufatura (PEGEMs): elementos-chave e modelo conceitual. Revista Gestão e Produção, v. 12, n. 3, p. 333-345, 2005. 
GREEN, P. E. J.; CARROLL, D.; GOLDBERG, S. M. A General Approach to Product Design Optimization Via Conjoint Analysis. Journal of Marketing, v. 45, n. 3, p. 17-37, 1981. http://dx.doi.org/10.2307/1251539

GUPTA, S.; ZEITHAML, V. Customer Metrics and Their Impact on Financial Performance. Marketing Science, v. 25, n. 6, p. 718-739, 2006. http://dx.doi.org/10.1287/ mksc. 1060.0221

HALLGREN, M.; OLHAGER, J. Quantification in manufacturing strategy: A methodology and illustration. International Journal of Production Economics, v. 104, n. 1, p. 113-124, 2006. http://dx.doi.org/10.1016/j. ijpe.2005.09.004

HAUSER, J. R. How Puritan-Bennett used the house of quality. Sloan Management Review, p. 61-70, 1993.

HAYES, R. et al. Produção, Estratégia e Tecnologia em Busca da Vantagem Competitiva. Porto Alegre: Artmed, 2008. 384 p.

HESS, J. D.; LUCAS, M. T. Doing the Right Thing or Doing the Thing Right: Allocating Resources Between Marketing Research and Manufacturing. Management Science, v. 50, n. 4, p. 521-26, 2004. http://dx.doi. org/10.1287/mnsc. 1030.0176

JAIN, D.; SINGH, S. S. Customer lifetime value research in marketing: A review and future directions. Journal of Interactive Marketing, v. 16, n. 2, p. 34-46, 2002. http://dx.doi.org/10.1002/dir.10032

LANCASTER, K. J. A New Approach to Consumer Theory. Journal of Political Economy, v. 74 n. 2, p. 132-157, 1966. http://dx.doi.org/10.1086/259131

LAW, A. M; KELTON, W. D. Simulation modeling and analysis. USA: McGraw Hill, 1991, 759 p.

MAlhotra, N. K. Pesquisa de Marketing uma Orientação Aplicada. 4. ed. Porto Alegre: Artmed, 2006. 720 p.

MATHAISEL, D. F. X. A lean architecture for transforming the aerospace maintenance, repair and overhaul (MRO) enterprise. International Journal of Productivity and Performance Management, v. 54, n. 8, p. 623-644, 2005. http://dx.doi.org/10.1108/17410400510627499 NEUMANN, J. von; MORGENSTERN, O. Theory of Games and Economic Behavior. 2th ed. Princeton: Princeton University Press, 1980. p. 648.

NIRAJ, R.; GUPTA, S.; NARASIMHAN, C. Customer profitability in a supply chain. Journal of Marketing, v. 65 , n. 3, p. 1-16, 2001. http://dx.doi.org/10.1509/ jmkg.65.3.1.18332

NUNnally, J. C.; BERnSTEIN, I. Psychometric Theory. 3th ed. McGraw-Hill Humanities/Social Sciences/Languages, 1994. 736 p.

PAIVA, E. L.; CARVALHO JUNIOR, J. M.; FENSTERSEIFER, J. E. Estratégia de Produção e de Operações: Conceitos, Melhores Práticas. Porto Alegre: Artmed, 2002. 192 p.

RIVERA, L.; CHEN, F. F. Measuring the impact of Lean tools on the cost-time investment of a product using cost-time profiles. Robotics and Computer-Integrated Manufacturing, v. 23, n. 6, p. 684-89, 2007. http:// dx.doi.org/10.1016/j.rcim.2007.02.013

RUST, R. T.; COOIL, B. Reliability measures for qualitative data: Theory and implication. Journal of Marketing Research, v. 31, n. 1, p. 1-14, 1994. http://dx.doi. org/10.2307/3151942

SAATY, T. L. Método de Análise Hierárquica. 2. ed. Rio de Janeiro: Makrom Books, 1991.

SLACK, N. Vantagem Competitiva em Manufatura. 2th ed. São Paulo: Atlas, 2002. 218 p.

TRAIN, K. Discrete Choice Methods with Simulations. Cambridge: University Press, 2003. 342 p. http://dx.doi. org/10.1017/CBO9780511753930

URBAN, G.; HAUSER, J. R. Design and Marketing of New Products. 2th ed. Prentice Hall, 1993. 701 p. 